\title{
Advanced Energy Detector Based Cooperative Spectrum Sensing In Cognitive Networks
}

\author{
Ritu $^{1}$ and Ameeta Seehra ${ }^{2}$ \\ ${ }^{1}$ Research Scholar, Electronics and Communication Department, Guru Nanak Dev Engineering College, Ludhiana, Punjab, India \\ ${ }^{2}$ Associate Professor, Electronics and Communication Department, Guru Nanak Dev Engineering College, Ludhiana, Punjab, India
}

\begin{abstract}
Cognitive radio is a befitting hopeful technology for future. Spectrum sensing is the most critical function of cognitive radio. Most of the spectrums sensing processes proposed earlier become impractical at lower signal to noise ratio conditions. Paper presents advanced cooperative spectrum sensing technique used in cognitive radio (CR) systems. By spectrum sensing it detects the presence of a primary user (PU) on a concerned spectrum. The accuracy of spectrum sensing depends on both sensing time and number of sensing nodes (secondary users) participating in the sensing process. We derive the optimal number of secondary users for cooperative spectrum sensing based on adaptive threshold energy detection to minimize spectrum detection error in conventional as well as improved energy detector.
\end{abstract}

\section{INTRODUCTION}

Wireless technology for communication has remarkably developed in last few decades. The cognitive radio is one another revolutionary enhancement of wireless technology. The rising congestion of spectrum and increase in demand of spectrum has left us searching for the alternates. Cognitive radio is sighted by the researchers as such an alternate. The cognitive radio continuously searches for the spectrum which is not in use by the Primary user for certain time slot. Cognitive radio hunts the vacant spectrum and uses it for secondary transmissions, so cognitive radios are also called SUs.Various sensing techniques can be applied at the sensing terminals of th CR. Some of the basic sensing techniques, which are commonly used are energy detection, cyclostationary method, matched filter detection and many more. In order to detect the primary signal using the matched filter, the secondary user need to have some prior knowledge of the Primary user's data signal. It is not possible all the times so matched filter based spectrum sensing is not always possible to be implemented. Similarly in cyclostationary feature extraction, cognitive radio searches for the features of detected signal similar to the priory known primary user signal. So for the same reason cyclostationary feature method can't be implemented flexibly.

So energy detection method best fits into the scenario where there is lack of sources to acquire the prior knowledge of the primary user signal. In a basic energy detector the energy of the received signal is computed as shown in the figure. The computed energy is then compared with a threshold level. Based on this comparison two hypothesis are followed $\mathrm{H} 0$ and $\mathrm{H} 1$. Here, H0 is followed when the energy detector interprets the presence of primary user as absent. $\mathrm{H} 1$ is followed when the presence of the primary user is interpreted as present.

\section{ENERGY DETECTOR MODEL}

Basic hypothesis model for transmitter detection

On the AWGN (Additive White Gaussian Noise) channels, the simplest spectrum sensing model is the binary hypothesis detect model.

$\mathrm{H} 0: \mathrm{Y}[\mathrm{N}]=\mathrm{W}[\mathrm{N}], \mathrm{H} 1: \mathrm{Y}[\mathrm{n}]=\mathrm{X}[\mathrm{n}]+\mathrm{W}[\mathrm{N}]$

Where $\mathrm{H} 0$ and H1express the different hypotheses of the primary users signal exist or not in the AWGN channels; $\mathrm{Y}[\mathrm{n}]$ is the signals received by the unlicensed users (cognitive users); $\mathrm{X}[\mathrm{n}]$ is the signals transmitted by the licensed users (primary users);

$\mathrm{W}[\mathrm{N}]$ is the White Gaussian Noise.According to the sensing algorithms, the statistic $\mathrm{T}$ can be obtained by $\mathrm{Y}[\mathrm{n}]$, and the sensing results can be obtained based on the decision theorem.

H0: $\mathrm{T}<\lambda$

$\mathrm{H} 1: \mathrm{T}>\lambda$

where $\lambda$ is the decision threshold.

Usually we use these two criteria to evaluate the spectrum detector's performance:

The probability of detection: $P d=P\{Y>\lambda \mid H 1\}$

The probability of false alarm: $P f=P\{Y>\lambda \mid$ Ho $\}$

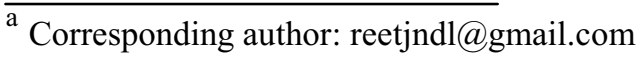


This work only focuses on the spectrum sensing in CR. where

Ho is the hypothesis of presence of primary user and $\mathrm{H} 1$ is the hypothesis of absence of primary user. False Alarm Probability Pf represents the case that SUs determines presence of PU but in fact PU is absent. Misdetection probability Pdian bankm represents the case that SUs determines absence of PU but in fact PU is using the network

Here $\tau$ is the detection time and $\omega$ is detection bandwidth. With these parameters mis-detection probability and false alarm probability are represented as follows:

$$
\begin{aligned}
& P m=P\{Y<\lambda \mid \mathrm{H} 1\}=1-Q \tau \omega\left((2 \lambda)^{\frac{1}{2}}, \lambda^{\frac{1}{2}}\right) \\
& P f=P\{Y>\lambda \mid \mathrm{H} 0\}=\frac{\Gamma\left(\tau \omega \frac{\lambda}{2}\right)}{\Gamma(\tau \omega)}
\end{aligned}
$$

where $\lambda$ is the energy threshold value, $Q \tau \omega$ is generalized Marcum Q Function, $\Gamma(\bullet)$ is gamma function, and $\Gamma(\bullet, \bullet)$ is incomplete gamma function respectively. $\mathrm{Pm}$ and $\mathrm{Pf}$ are functions of parameters $\lambda, \tau$, and $\omega$.

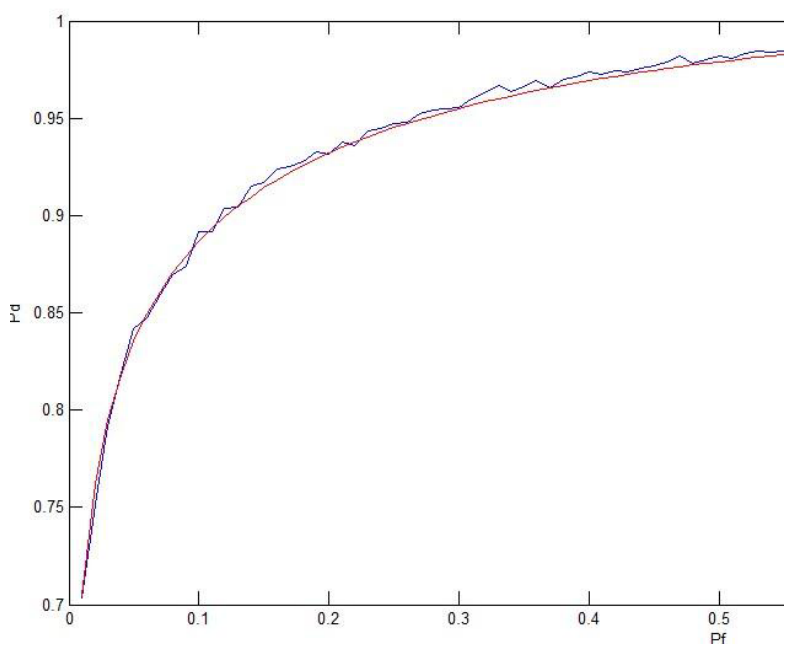

Figure 1. Energy detector ROC.

There are still many loops and holes in energy detector based spectrum sensing. Firstly, the energy detectors performance degrades at the lower SNR values. Secondly, the selection of the threshold is very crucial for the performance of the energy detector. Estimating the threshold is a provocative issue. The threshold should be such chosen so that it does not favour any of decision hypothesis. . Thirdly, the shadowing problem overshadows the primary user which leads to the unfit decisions. Lastly, the single cognitive radio sensing is least reliable. The wrong decisions will ultimately cause higher interference to the Primary user. So requirement in spectrum sensing is to make the sensing most reliable and hence to reduce the probability of interference to the Primary user.

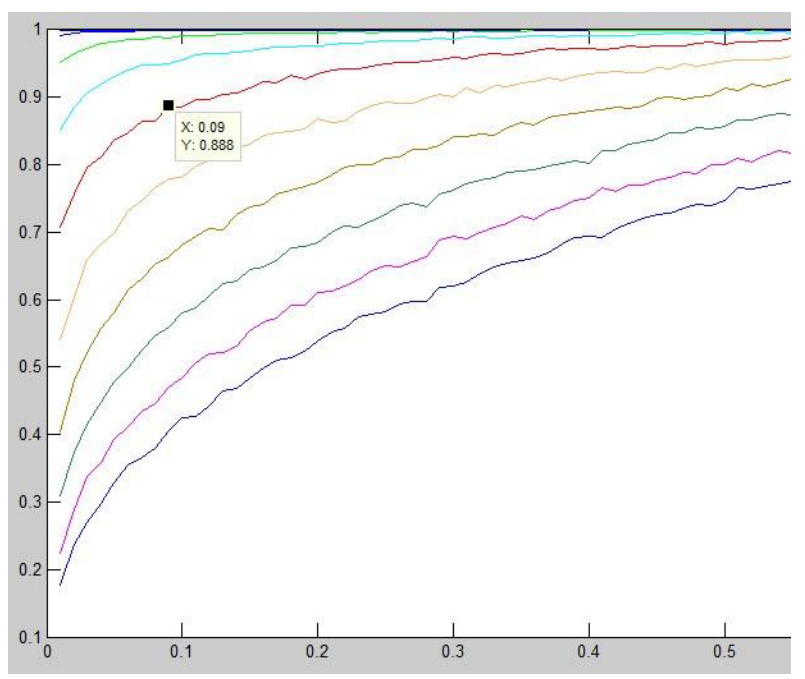

Figure 2. Energy detector ROC with adaptive threshold

Fig 2 shows the ROC of energy detector with adaptive threshold. Here threshold varies with change in the SNR value. This means the noise level directly effects the sensing performance of the detector.

\section{ADVANCED ENERGY DETECTOR}

In conventional energy detector decision is made after comparison to single threshold. In another proposed work double threshold detection is used. Instead of two decision regions there are three. Third one is no decision area. Here CR is not sure so it just does not contribute to the decision. The decision hypothesis followed will be

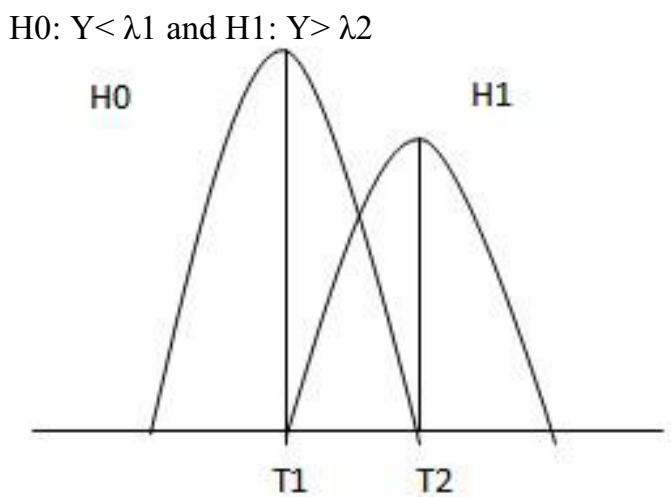

Figure 3. Double threshold decision hypothesis

The probability of detection and probability of false alarm for double threshold detector can be defined asThe probability of detection:

$\mathrm{Pd}=\mathrm{P}\{\mathrm{y}>\lambda 2 \mid \mathrm{H} 1\}$

The probability of false alarm: $\mathrm{Pf}=\mathrm{P}\{\mathrm{y}>\lambda 2 \mid \mathrm{H} 0\}$ 


\section{COOPERATIVE SPECTRUM SENSING}

Cooperative spectrum sensing schemes are categorized by the way to determine the presence of primary user (PU) with the collected information from secondary users (SUs) at a fusion center. The OR-rule declares presence of PU as long as at least one SU detects the PU. With the assumption of number of $\mathrm{N}$ SUs, the mis-detection probability and false alarm probability at a fusion centre are computed from equation

OR Rule : $\left\{\begin{array}{l}P_{m, t}=P_{m}{ }^{N} \\ P_{f, t}=1-\left(1-P_{f}\right)^{N}\end{array}\right.$

where $\mathrm{Pm}, \mathrm{t}$ is the mis-detection probability and $\mathrm{Pf}, \mathrm{t}$ is the false alarm probability at a fusion centre whereas $\mathrm{Pm}$ and $\mathrm{Pf}$ are the misdetection and false alarm probability determined by each secondary user. The AND-rule declares presence of PU only when all SUs recount the PU's presence. For the AND-rule the mis-detection probability and false alarm probability are computed as follows:

$$
\text { AND Rule : }\left\{\begin{array}{l}
P_{m, t}=1-\left(1-P_{m}\right)^{N} \\
P_{f, t}=P_{f} N
\end{array}\right.
$$

The majority-rule declares the presence of PU when more than half of SUs report presence of PU. The majority rule has characteristic of OR-rule and AND-rule and its misdetection probability and false alarm probability are derived as:

Total detection error probability can be defined as the average of mis-detection probability and false alarm probability by each hypothesis probability.

$\mathrm{Pe}, \mathrm{t}=\mathrm{P}(\mathrm{H} 1) \mathrm{Pm}, \mathrm{t}+\mathrm{P}(\mathrm{H} 0) \mathrm{Pf}, \mathrm{t}$

Our goals to find Kopt for K-out-of-N-rule which minimizesthe total detection error probability. From (11) and (12) the value obtained for $\mathrm{K}$ optimal is :

$$
\begin{aligned}
& \text { Kout of N } \\
& \text { rule : }
\end{aligned}\left\{\begin{array}{c}
\text { Pm, }=1-\sum_{j=K}^{N}\left(\begin{array}{c}
N \\
j
\end{array}\right)(1-\mathrm{Pm})^{j} P m^{N-j} \\
\mathrm{Pf}, \mathrm{t}=\sum_{j=K}^{N}\left(\begin{array}{l}
N \\
j
\end{array}\right)(1-\mathrm{Pf})^{N-j} P f^{j}
\end{array}\right.
$$

This gives the expression for optimal value of number of CUs required for the decision which gives minimum error probability. The total error is sum of $\mathrm{Pm}$ and $\mathrm{Pf}$ $(\mathrm{P}(\mathrm{H} 1)=\mathrm{P}(\mathrm{H} 0)=0.5)$ which should be least. The value of $\mathrm{K}$ optimal can be calculated by finding the minimum error value.[7]
In conventional energy detection technique single threshold energy detector has been used. Advanced scheme has been implemented in cooperative manner to find the optimal number of CRs required, total error rate.

\section{RESULTS AND DISCUSSION}

The conventional energy detector based cooperative environment was created and simulated. Total CRs present over the network are 20. The optimal number of CRs comes out to be 15 , where the minimum error is calculated

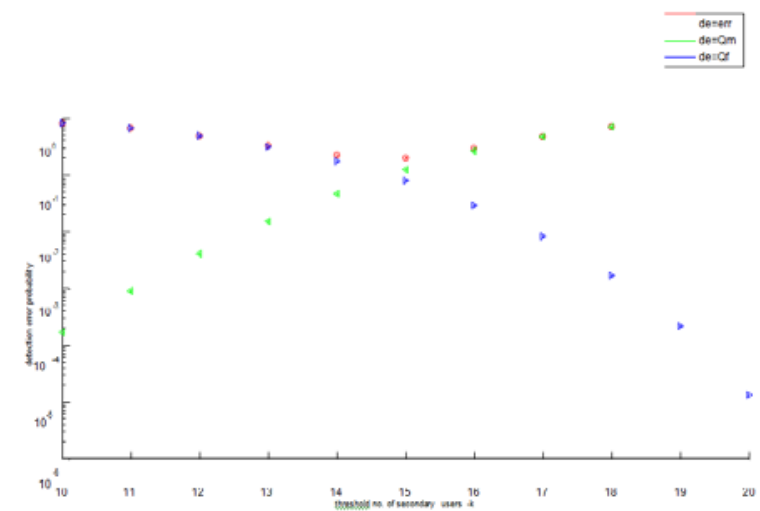

Figure 4. Three detection error probabilities (total detection error probability $\mathrm{Pe}$, false alarm probability $\mathrm{Pf}$, mis-detection probability $\mathrm{Pm}$ )

In another simulation the dual threshold based energy detector is compared to conventional one. The results are shown in figure 5 and 6.

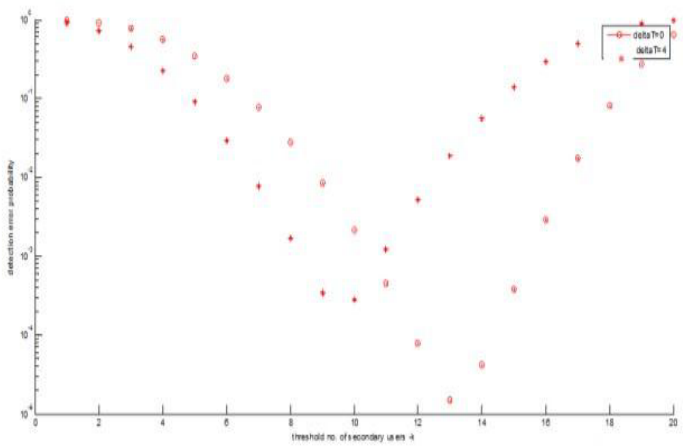

Figure 5. Error to number of CRs in conventional Vs. Double

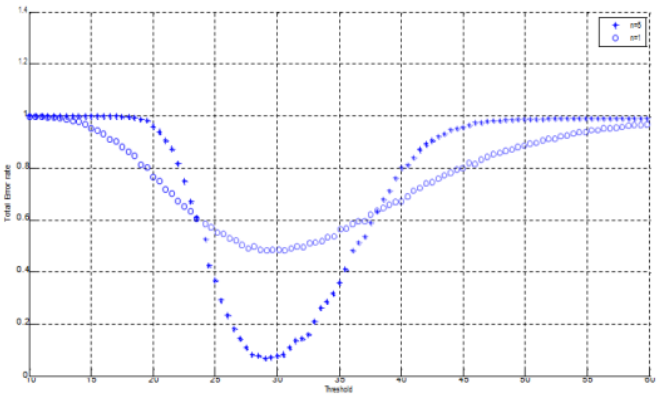

Figure 6. Total error Vs threshold for single CR and cooperative sensing for $\Delta \mathrm{T}=8$ 

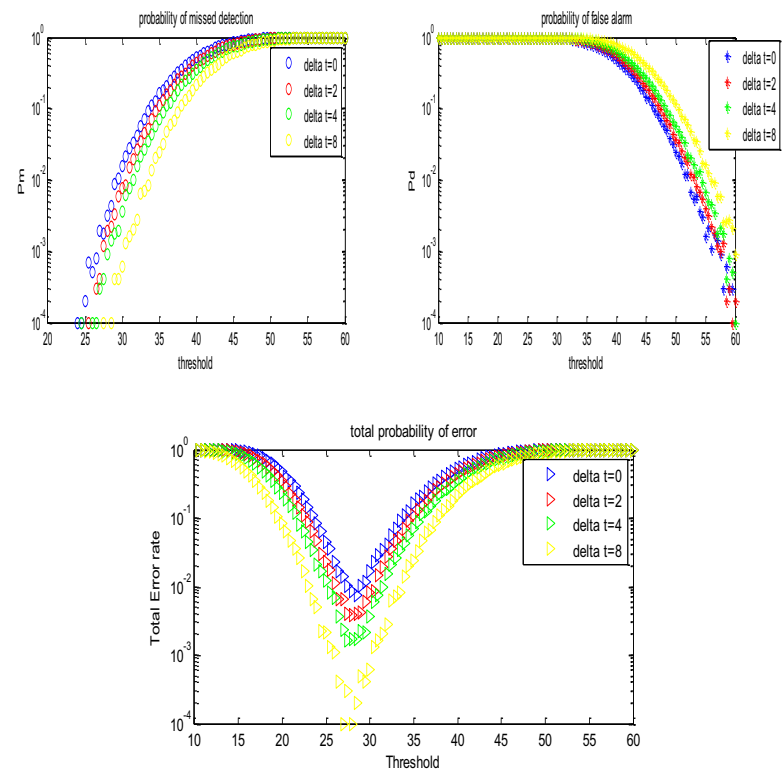

Figure 7. Pm, Pd and Pe for different threshold difference in double threshold detector

Double threshold ED is evaluated for different values of threshold difference. Figure. 7 shows $\mathrm{P}_{\mathrm{m}}, \mathrm{P}_{\mathrm{d}}$ and $\mathrm{P}_{\mathrm{e}}$ with respect to threshold difference in double threshold energy detector. It is to be noted here that the minimum $\mathrm{P}_{\mathrm{m}}$ is corresponding to maximum $\Delta \mathrm{T}$ i.e. 8 in this case. $\mathrm{P}_{\mathrm{m}}$ reduces with decrease in threshold. $P_{f}$ reduces as the threshold value increases. The total error $P_{e}$ first decreases and then increases with increase in threshold. Four cases of different threshold differences can be seen here. The curves corresponding to $\Delta \mathrm{T}=0$ is single threshold ED curve.

\section{CONCLUSION AND FUTURE SCOPE}

The OR-rule has very low misdetection probability which is helpful to protect the primary user. In the meantime it has relatively high false alarm probability which makes the spectrum usage inefficient. The AND-rule has very low false alarm which makes spectrum usage efficient, however it may not protect the primary user from strong interferences from the secondary users. As a result the quality of services for primary user is not guaranteed Larger K usually increases misdetection probability but decreases false alarm probability. When misdetection probability is very low larger $\mathrm{K}$ is better to reduce false alarm probability. Thus cooperative sensing can be used to reduce the error rate; hence interference to PU can also be reduced. Single CR gives the Pe .99 and in case of optimal number of CRs with cooperation it reduces to 0.009 . In conventional energy detector based cooperative network lesser number of CRs are required but error is more, whereas optimal CRs required are more in advanced network and error is lesser. Error can be considerably reduced by increasing the threshold difference. Further advancement can be made by varying threshold.

\section{References}

1. S. Ziafat, W. Ejaz, and H. U. Jamal "spectrum sensing techniques for cognitive radio networks: performance analysis" International Microwave Workshop Series on Intelligent Radio for Future Personal Terminals, pp. 1-4, (2011).

2. T. Yucek and H. Arslan, "A Survey of Spectrum Sensing Algorithms for Cognitive Radio Applications," IEEE communications surveys \& tutorials, vol. 11, no. 1, (2009).

3. Y. Shi, J. Guo and Y. Jian, "Spectrum Sensing Algorithms in the Cognitive Radio Network," Emerging Intelligent Computing Technology and Applications, vol. 304, pp. 132-138, (2012).

4. D. Cabric, S. M. Mishra and R. W. Brodersen "Implementation Issues in Spectrum Sensing for Cognitive Radios," in Proc. 38th Asilomar Conference on Signals, Systems and Computers, pp. 772776, (2004).

5. Y.C. Liang, Y. Zeng, E.C.Y. Peh, and A. T. Hoang, "Sensing-Throughput Tradeoff for Cognitive Radio Networks," IEEE Transactions On Wireless Communications, Vol. 7, No. 4, pp 1326-1338, (2008).

6. G. Ganesan and Y Li, "Cooperative Spectrum Sensing in Cognitive Radio,Part I: Two User Networks," IEEE Transactions On Wireless Communications, vol. 6, pp. 2204-2214, (2007).

7. C. You, H. Kwon, and J. Heo, "cooperative TV spectrum sensing in cognitive radio for Wi-Fi networks" IEEE transactions on consumer electronics, vol. 57 , ( 2011).

8. A. Satheesh, S. H. Aswini, S. G. Lekshmi, S. Sagar, "Spectrum sensing techniques A comparison between energy detector and cyclostationarity detector", International Conference on Control communication computing, pp: 393, (2013). 